\title{
ABSOLUTE ENDPOINTS OF CHAINABLE CONTINUA
}

\author{
IRA ROSENHOLTZ
}

(Communicated by Doug W. Curtis)

\begin{abstract}
An endpoint of chainable continuum is a point at which it is always possible to start chaining that continuum. Some endpoints appear to have the property that one is almost "forced" to start (or finish) the chaining at these points. This paper characterizes these "absolute endpoints", and this characterization is used to show that in a chainable continuum locally connected at $p$ is equivalent to connected im kleinen at $p$.
\end{abstract}

Introduction. Roughly speaking, an endpoint of a chainable continuum is a point at which it is always possible to start chaining that continuum. R. H. Bing used this notion to study chainable continua in general and the pseudo-arc in particular in several of his landmark papers $[2,3,4]$. Some endpoints of chainable continua appear to have the additional property that, not only is it possible to start chaining there, but that a person is practically "forced" to start (or finish) the chaining at those points. The main purpose of this paper is to characterize these "absolute endpoints". Along the way, we will use their characterization to show that, in a chainable continuum, locally connected at $p$ is equivalent to connected im kleinen at $p$. This answers a question R. H. Bing asked me in conversation. This paper is affectionately dedicated to the memory of Professor Bing.

Preliminaries. A continuum is a compact connected metric space. The statement that a continuum $X$ is chainable means that, for each positive real number $\varepsilon, X$ possesses a finite cover consisting of open subsets $C_{1}, C_{2}, \ldots, C_{n}$ of $X$, each having diameter less than $\varepsilon$, with the property that $C_{i}$ intersects $C_{j}$ if and only if $j=i-1, j=i$, or $j=i+1$. (Loosely speaking, $X$ must have open covers consisting of small sets which fit together "like a chain".) Such an open cover with be called an $\varepsilon$-chain, and the $C_{j}$ 's will be referred to as links. (See Figure 1.) For example, an arc, a topologist's sine curve, and a pseudo-arc (see $[\mathbf{2}, \mathbf{6}]$ ) are chainable, while a simple closed curve and the letter " $T$ " are not.

Certain fairly "well known" properties of chainable continua will be assumed, without proof. However, to make these more meaningful, some vocabulary would be helpful.

Suppose that $X$ is a continuum.

The statement that $X$ is unicoherent means that $X$ is not the union of two subcontinua whose intersection is disconnected. For example, a simple closed curve is not unicoherent, but an arc is.

Received by the editors October 30, 1986 and, in revised form, April 16, 1987.

1980 Mathematics Subject ('lassification. Primary 54F 15, 54F20, 54F50, 54D05.

Key words and phrases. Chainable continua, endpoints, connectedness im kleinen, local connectedness.

(C) 1988 American Mathematical Society $0002-9939 / 88 \$ 1.00+\$ .25$ per page 


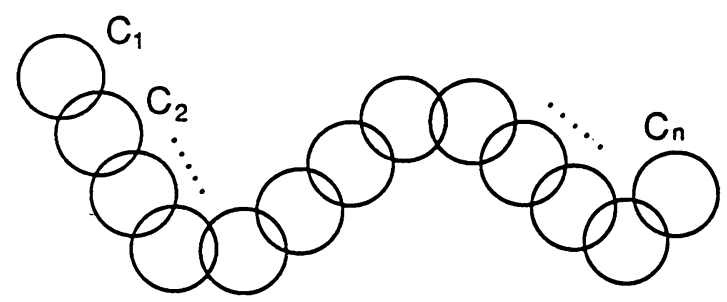

FIGURE 1

The statement that $X$ is a triod means that $X$ contains a subcontinuum whose complement is the union of three nonempty pairwise disjoint open sets. $X$ is atriodic if and only if it contains no subcontinuum which is itself a triod. For example, the letter " $T$ " is a triod, and a topologist's sine curve is atriodic.

If $p$ and $q$ are points of $X$, and no proper subcontinuum of $X$ contains both $p$ and $q$, then $X$ is irreducible between $p$ and $q$. If $X$ is irreducible between some pair of its points, then $X$ is called irreducible. For example, an arc is irreducible between its endpoints, and is therefore irreducible, while a simple closed curve and the letter " $T$ " are not.

The relevance of the above definitions to chainable continua is contained in the following "well-known" result(s).

THEOREM 0.0. If $X$ is a chainable continuum, then each subcontinuum of $X$ is chainable, unicoherent, atriodic, and irreducible.

Here are a few more useful definitions.

The statement that the continuum $X$ is decomposable means that $X$ is the union of two proper subcontinua. If $X$ is nondegenerate and is not decomposable, it is called, not surprisingly, indecomposable. An arc is decomposable and a pseudoarc indecomposable. (In fact, a pseudo-arc is a chainable continuum, each subcontinuum of which is indecomposable-and this describes the pseudo-arc up to homeomorphism (see [3])).

If $X$ is a continuum and $p$ is a point of $X$, then the composant of $X$ belonging to $p$ is defined to be the union of all proper subcontinua of $X$ which contain $p$. A set is a composant of $X$ provided it is the composant of $X$ belonging to $p$, for some point $p$ of $X$. For example, an arc has exactly three distinct composants.

Finally, it will be convenient to refer to the following brother-and-sister theorems of general topology by name.

TheOREM 0.1 (The SplitTing TheOREM). Suppose that $H$ and $K$ are disjoint nonempty closed subsets of the compact metric space $M$, and suppose further that no continuum of $M$ intersects both $H$ and $K$. Then $M$ is the union of two disjoint closed (and open) sets, one containing $H$, the other containing $K$.

ThEOREM 0.2 (The TO-THE-Boundary THEOREM). Suppose that $X$ is a continuum, and $U$ is a proper open subset of $X$. Then each component of $U$ has at least one limit point on the boundary of $U$.

For proofs of these theorems, see [5 and 7].

We may now proceed. 
ABSOLUTE ENDPOINTS. In his paper Snake-like continua [4], R. H. Bing proved the equivalence of the following conditions on a chainable continuum $X$ and a point $p$ of $X$ :

(A) Each nondegenerate subcontinuum of $X$ containing $p$ is irreducible from $p$ to some other point.

(B) If each of two subcontinua of $X$ contains $p$, then one of the subcontinua contains the other.

(C) For each positive number $\varepsilon$, there is an $\varepsilon$-chain of $X$ such that only the first link contains $p$.

A point $p$ of a chainable continuum $X$ is called an endpoint of $X$ if $p$ and $X$ satisfy any of the conditions above. Thus, an arc has two endpoints and a topologist's sine curve has three endpoints. The pseudo-arc has a fantastic property that it is chainable and all of its points are endpoints, and again this characterizes the pseuod-arc.

Some endpoints of chainable continua appear to have the property that, not only is it possible to start chaining there, but that a person is practically "forced" to start (or finish) the chaining at these points.

We now endeavor to characterize these "absolute endpoints". This problem was suggested to me by Professor Joseph M. Martin.

Before proceeding with the characterization, I think it is instructive to look at a notion of "absolute endpoint" which does not work.

THEOREM MINUS ONE. Suppose that $X$ is a chainable continuum and $p$ is a point of $X$. One might wish to call $p$ an "absolute endpoint" if there is a positive number $\varepsilon$ such that each $\varepsilon$-chain of $X$ contains $p$ in its first or last link.

However, with this "definition", no nondegenerate chainable continuum ever has absolute endpoints.

IndiCATION OF PROOF. Assume that $X$ is a nondegenerate chainable continuum, $p$ is a point of $X$, and $\varepsilon$ is a positive real number such that each $\varepsilon$-chain of

A Close-up:

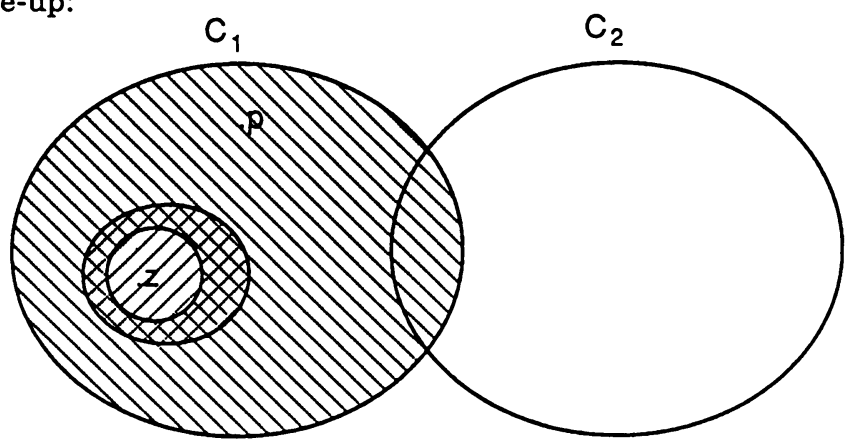

shades new first link

shades new second link

FIGURE 2 
$X$ contains $p$ in its first or last link. Let $C_{1}, \ldots, C_{n}$ be an $\varepsilon$-chain of $X$ with $p$ belonging to $C_{1}$. We may assume that $p$ is not an element of $\bar{C}_{2}$. (Otherwise, we could have started with an $\varepsilon / 2$-chain of $X$ with more than two links, and with $p$ in the first link, and then lump the first two links together to get an $\varepsilon$-chain with this property.) Let $z$ be a point of $C_{1}-\bar{C}_{2}$ different from $p$, and now "eat away" at $C_{1}-\bar{C}_{2}$ to construct a new $\varepsilon$-chain with $p$ not in the first or last link. (See Figure 2.) (In fact, if we wish, we can place $p$ in the "middle link" by repeating this process.) This is a contradiction.

REMARK. The characterization we finally obtain shows that this "eating away" process is, in some sense, the only flaw in the above attempt at defining these "absolute endpoints".

We now state the main theorem of this paper.

THEOREM 1.0 (THE CHARACTERIZATION). Suppose $X$ is a chainable continuum and $p$ is a point of $X$. Then the following conditions are equivalent:

(1) If $X$ is irreducible between $x$ and $y$, then either $x$ or $y$ is $p$.

(2) $X-\{p\}$ is a composant of $X$.

(3) $X$ is irreducible between $p$ and some other point, and $X$ is connected im kleinen at $p$.

(4) $X$ is irreducible between $p$ and some other point, and $X$ is locally connected at $p$.

(5) $X$ is locally connected at $p$, and $p$ does not separate $X$.

(6) $X$ is locally connected at $p$, and whenever $C_{1}, \ldots, C_{n}$ is an $\varepsilon$-chain of $X$ with $p$ belonging to a connected link $C_{k}$, then either: (a) $k=1$, (b) $k=n$, (c) $k=2$ and $\bar{C}_{2}$ contains $C_{1}$, or $(\mathrm{d}) k=n-1$ and $\bar{C}_{n-1}$ contains $C_{n}$.

(7) For each positive number $\varepsilon$, there is a positive number $\delta$ such that, if $C_{1}, \ldots$, $C_{n}$ is a $\delta$-chain of $X$ and $p$ belongs to $C_{k}$, then either $\bigcup_{j=1}^{k} C_{j}$ or $\bigcup_{j=k}^{n} C_{j}$ is contained in an $\varepsilon$-neighborhood of $p$.

And finally, each of (1) through (7) being true implies the truth of the corresponding statement for each subcontinuum containing $p$.

(Note. A continuum $X$ is connected im kleinen at the point $p$ if and only if for each open set $U$ of $X$ containing $p, p$ lies in the interior of a connected subset of $U$.

Before proceeding with the details of the proof, it is worth remarking that the following result of Lida K. Barrett implies that these so-called "absolute endpoints" actually exist in profusion. For a proof, see [1].

THEOREM 1.1. If $X$ is a chainable continuum, each subcontinuum of which is decomposable, then $X$ contains a subcontinuum $X^{\prime}$ which is irreducible between a unique pair of points.

We procrastinate further and state a useful lemma. The proof is left to the reader.

LEMMA 1.2. If $X$ is any continuum (not necessarily chainable), then the following are equivalent:

(a) Each subcontinuum of $X$ is unicoherent.

(b) The intersection of any collection of subcontinua of $X$ is connected. 
(c) Each two points of $X$ belong to a unique subcontinuum which is irreducible between them.

Notation. If $X$ is chainable, and $x$ and $y$ are points of $X$, then (by Theorem 0.0 and Lemma 1.2) there is a unique subcontinuum of $X$ which is irreducible between $x$ and $y$. We shall denote this continuum by $K_{x y}$.

We are now ready to prove Theorem 1.0. In what follows, $X$ is a chainable continuum, $p$ is the point of $X$ under observation, and the "conditions" refer to the conditions of Theorem 1.0.

\section{PROPOSITION 1.3. Conditions (1)-(4) are equivalent.}

PrOOF. Condition (4) implies condition (3): This is trivial.

Condition (3) implies condition (2): Suppose $X$ is irreducible between $p$ and $x$, and that $X$ is connected im kleinen at $p$. Let $L$ be a "small" continuum containing $p$ in its interior and not containing $x$. By irreducibility, it follows that $X-L$ is connected, and thus the closure of $X-L$ is a continuum containing $x$ and not $p$. Since $L$ can be chosen to be arbitrarily "small" by connectedness im kleinen at $p$, this shows that $X-\{p\}$ is a composant.

Condition (2) implies condition (1): Suppose $X-\{p\}$ is the composant of $X$ belonging to $q$, and suppose that $X$ is irreducible between $x$ and $y$. If $x$ and $y$ are both not $p$, then, by condition (2), $K_{x q}$ and $K_{y q}$ are proper subcontinua, in fact not containing $p$. But then $X=K_{x y}=K_{x q} \cup K_{y q}$ is contained in $X-\{p\}$. This contradiction implies that $x$ or $y$ must have been $p$.

Condition (1) implies condition (4): Assume that Condition (1) holds. Since $X$ is chainable, and therefore irreducible, it follows immediately that $X=K_{p x}$ for some $x$.

Now let $W$ be $\bigcap\{L \mid L$ is a continuum in $X$ and $p \in \operatorname{Int} L\}$. We wish to show that $W=\{p\}$. By way of contradiction, suppose that there is a point $q$ in $W$ which is different from $p$. Now $X=K_{p q} \cup K_{q x}$, and hence by (1), $p \in X-K_{q x}$. But $X-K_{q x}$ is open in $X$ and is contained in $K_{p q}$. Hence $p \in \operatorname{Int} K_{p q}$, and so $W$ is contained in $K_{p q}$. Now since $p$ and $q$ are both points of $W$ and $W$ is connected, it follows that $K_{p q}$ is contained in $W$, and hence $W=K_{p q}$. We have shown that if $q$ is any point of $W$ different from $p$, then $W=K_{p q}$. So $W$ has no nondegenerate proper subcontinua containing $p$. But this contradicts the To-the-Boundary Theorem, and so we must conclude that $W=\{p\}$.

Finally, to show that $X$ is locally connected at $p$, suppose that $U$ is a given open subset of $X$ containing $p$. Then $U$ contains $W$, and by the definition of $W$ and compactness, we see that there is a finite collection of continua $L_{1}, \ldots, L_{n}$ of $X$ such that $\bigcap_{j=1}^{n} L_{j}$ is contained in $U$, and, for each $j, p \in \operatorname{Int} L_{j}$. Now because $X$ is irreducible between $p$ and some other point, the interior of any continuum containing $p$ is connected. And so $p$ belongs to a connected open set which is contained in $U$, namely $\operatorname{Int}\left(\bigcap_{j=1}^{n} L_{j}\right)$. This argument implies that $X$ is locally connected at $p$.

REMARK (AN ASIDE). A theorem about chainable continua probably should not be given a "cyclic proof", as above. However, in this case, an indignant reader may take solace from the fact that one of the "links" of the proof was "trivial".

We are now ready to "make it official". 
DEFINITION. A point $p$ of a chainable continuum $X$ is an absolute endpoint of $X$ if $p$ and $X$ satisfy any of the conditions of Proposition 1.3.

REMARK. Observe that if $Y$ is a subcontinuum of $X$ and $x$ and $y$ are points of $Y$, then the continuum of $Y$ irreducible between $x$ and $y$ is the same as the continuum of $X$ irreducible between $x$ and $y$. From the observation, it follows that condition (1) implies the following a priori stronger condition:

$\left(1^{\prime}\right)$ If $Y$ is a subcontinuum of $X$ containing $p$, and $Y$ is irreducible between $x$ and $y$, then either $x$ or $y$ is $p$.

Thus, if $p$ is an absolute endpoint of $X$, then $p$ is also an absolute endpoint of each subcontinuum of $X$ containing $p$. (In particular, if $p$ is an absolute endpoint of $X$, then each subcontinuum of $X$ containing $p$ is decomposable.) In addition, this observation and Bing's theorem imply that absolute endpoints are indeed endpoints. This last fact should become even clearer, as we progress with the proof of Theorem 1.0 .

Now, back to the proof of Theorem 1.0.

LEMMA 1.4. Suppose that $p$ is a point of the chainable continuum $X$, and that $X$ is connected im kleinen at $p$. Then either $p$ is an absolute endpoint of $X$, or $X$ is the union of two subcontinua having only $p$ in common and each having $p$ as absolute endpoint. In the latter case, $p$ separates $X$.

PROOF. If $X$ is irreducible between $p$ and some other point, then $p$ is an absolute endpoint of $X$. If not, then there are points $x$ and $y$ of $X$, both different from $p$, so that $X=K_{x y}$. But then $X=K_{x p} \cup K_{y p}$. Now $K_{x p}$ (and similarly, $K_{y p}$ ) is a continuum irreducible between $p$ and some other point, and by unicoherence and our hypothesis, is connected im kleinen at $p$. So $K_{x p}$ has $p$ as absolute endpoint.

We wish to show that $K_{x p} \cap K_{y p}=\{p\}$. Certainly this intersection is a continuum containing $p$, so since $p$ is an absolute endpoint of $K_{x p}$, there is a point $z$ such that $K_{x p} \cap K_{y p}=K_{z p}$. If $z=p$, then $K_{z p}=\{p\}$, and we are done. So by way of contradiction, assume $z$ is not $p$. Now $X=K_{x y}=K_{x z} \cup K_{y z}$, so $p$ belongs to $K_{x z}$ or $K_{y z}$. We will assume that the notation is chosen so that $p$ belongs to $K_{x z}$. Then $K_{x z}=K_{x p} \cup K_{z p}$. But since $K_{z p}$ is contained in $K_{x p}$, this implies that $K_{x z}=K_{x p}$. However, $p$ is an absolute endpoint of $K_{x p}$, so $x$ or $z$ must be $p$, a contradiction.

The final assertion follows from the following: $X-\{p\}=\left(K_{x p} \cup K_{y p}\right)-\{p\}=$ $\left(K_{x p}-\{p\}\right) \cup\left(K_{y p}-\{p\}\right)$ a separation.

\section{PROPOSITION 1.5. Condition (1) and condition (5) are equivalent.}

This follows directly from Lemma 1.4 and Proposition 1.3.

COROLLARY 1.6. If $X$ is a chainable continuum and $X$ is locally connected at $p$, then for each positive number $\varepsilon$, there is an $\varepsilon$-chain of $X$ with $p$ in a connected link.

Proof. If $p$ is an absolute endpoint of $X$, then it is an endpoint. So chain $X$ with $p$ in oniy the first link, and then "eat away" at the first link as in the proof of Theorem Minus One-except that, instead of eating near $z$, take a "connected bite" around $p$.

If $p$ is not an absolute endpoint of $X$, then by Lemma $1.4, X$ is the union of two subcontinua having only $p$ in common and each having $p$ as absolute endpoint. 
Thus, each half is locally connected at $p$, and as above we may chain each half with $p$ belonging only to the connected first links. Put the chains together to form a chain of $X$ with $p$ in a connected link.

The next theorem now follows easily from Proposition 1.3, Lemma 1.4, and the proof of Corollary 1.6.

THEOREM 1.7. In a chainable continuum, locally connected at $p$ is equivalent to connected im kleinen at $p$.

Continuing with the proof of Theorem 1.0, we prove the following:

Proposition 1.8. Condition (4) is equivalent to condition (6).

ProOF. Condition (4) implies condition (6): Suppose $X$ is irreducible between $p$ and some other point. By way of contradiction, suppose that $C_{1}, C_{2}, \ldots, C_{n}$ is an $\varepsilon$-chain of $X, p$ is in the connected link $C_{k}$, and that none of the conclusions $a, b, c$, or $d$ holds. Then since $\bar{C}_{k}$ is contained in $C_{k-1} \cup C_{k} \cup C_{k+1}$, this implies that $\bar{C}_{k}$ separates $X$. However, from this separation and the fact that $\bar{C}_{k}$ is connected, it follows that $X$ is not irreducible between $p$ and any other point, a contradiction.

Condition (6) implies condition (4): Since $X$ is locally connected at $p$, Corollary 1.6 implies that $X$ can be chained with $p$ in a connected link. By condition (6), this link must be one of the first or last two. From this it is clear that $p$ is an endpoint, and so, by Bing's theorem, $X$ is irreducible from $p$ to some other point.

We will complete the proof of Theorem 1.0 by showing that condition (5) implies condition (7), and that condition (7) implies condition (1). But first, a lemma.

LEMMA 1.9. Let $X$ be a chainable continuum. Suppose $H_{1}$ and $H_{2}$ are subcontinua of $X$ with $H_{1}$ containing $H_{2}$, and that each of $H_{1}$ and $H_{2}$ separates $X$. For $j=1,2$, let $X-H_{j}=L_{j} \cup R_{j}$ be given separations.

Then, by possibly interchanging $L_{2}$ for $R_{2}$ and vice versa, the notation can be chosen so that $L_{1}$ is contained in $L_{2}$, and $R_{1}$ is contained in $R_{2}$.

ProOF. First note that each $L_{j}$ and $R_{j}$ must be connected (otherwise $X$ is a triod), so $L_{1} \subseteq X-H_{1} \subseteq X-H_{2}=L_{2} \cup R_{2}$, a separation, gives us that either $L_{1}$ is contained in $L_{2}$ or $R_{2}$, and we assume $L_{1} \subseteq L_{2}$. Similarly, $R_{1}$ is contained in $L_{2}$ or $R_{2}$, and we must show that $R_{1} \subseteq R_{2}$. (See Figure 3.)

Notice that $X-R_{2}=H_{2} \cup L_{2}$, a continuum, so by unicoherence, $H_{1}-R_{2}=H_{1} \cap$ $\left(X-R_{2}\right)$ is also a continuum. Now, if we were to assume, by way of contradiction, that $R_{1} \subseteq L_{2}$, then $X-\left(H_{1}-R_{2}\right)=R_{2} \cup L_{1} \cup R_{1}$ would be a separation, implying that $X$ is a triod. This is impossible since $X$ is chainable, so we must conclude that $R_{1} \subseteq R_{2}$.

\section{PROPOSITION 1.10. Condition (5) implies condition (7).}

PROOF. Suppose, by way of contradiction, that there is a positive number $\varepsilon$ such that: for each positive number $\delta$ there is a $\delta$-chain $C_{1}, \ldots, C_{n}$ of $X$ such that $p \in C_{k}$ and both $\bigcup_{j=1}^{k} C_{j}$ and $\bigcup_{j=k}^{n} C_{j}$ get at least as far as $\varepsilon$ from $p$. In an $\varepsilon / 2$-neighborhood of $p$, let $U$ be a connected open set containing $p$ (this is possible since we are assuming that $X$ is locally connected at $p$ ). Let $\delta$ be one quarter of the distance from $p$ to the complement of $U$, and let $C_{1}, \ldots, C_{n}$ be a specific $\delta$-chain of $X$ with $p \in C_{k}$ and both $\bigcup_{j=1}^{k} C_{j}$ and $\bigcup_{j=k}^{n} C_{j}$ containing points at least as far as 


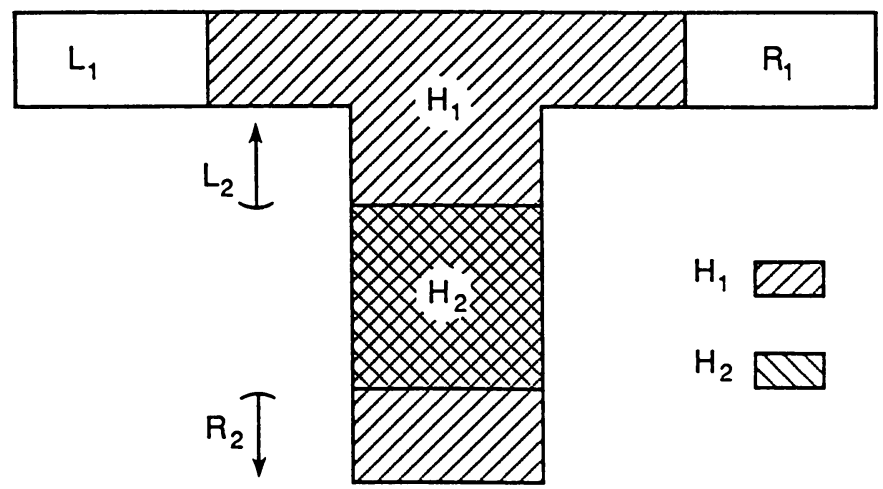

FIGURE 3

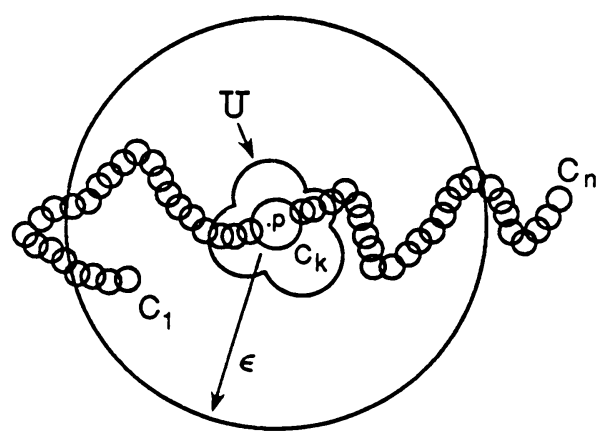

FIGURE 4

$\varepsilon$ from $p$. Notice that $\bar{U}$ separates $X\left(X-\bar{C}_{k}\right.$ is the union of two disjoint open sets, each of which intersects $X-\bar{U}$, and $X-\bar{C}_{k}$ contains $\left.X-\bar{U}\right)$, say $X-\bar{U}=L \cup R$ (for left and right). (See Figure 4.)

Repeating this procedure again and again, we can obtain a sequence of connected open sets $U_{1} \supseteq U_{2} \supseteq \cdots$ each containing $p$, so that $\{p\}=\bigcap_{j=1} \bar{U}_{j}$, and each $\bar{U}_{j}$ separates $X$ as $X-\bar{U}_{j}=L_{j} \cup R_{j}$. By the preceding lemma, we may assume $L_{1} \subseteq L_{2} \subseteq L_{3} \subseteq \cdots$ and $R_{1} \subseteq R_{2} \subseteq R_{3} \subseteq \cdots$. But then $X-\{p\}=\left(\bigcup_{j=1}^{\infty} L_{j}\right) \cup$ $\left(\bigcup_{j=1}^{\infty} R_{j}\right)$, a union of disjoint open sets. So $p$ separates $X$, a contradiction.

REMARK. We have actually proved the following fact: In a chainable continuum, if each of a decreasing collection of subcontinua separates, then so does their intersection. Shorter proofs, using previously known equivalences, were abandoned here for this reason.

PROPOSITION 1.11. Condition (7) implies condition (1).

PrOOF. This will be proven in several steps.

Step 1. Suppose condition (7) is satisfied. For a fixed positive number $\varepsilon$, let $\delta$ be a positive number as guaranteed by condition 7 . Suppose $D_{1}, \ldots, D_{u}$ is a $\delta$-chain of $X, p \in D_{k}$, and $\bigcup_{j=1}^{k} D_{j}$ is contained in $B(p, \varepsilon)$, the $\varepsilon$-ball about $p$. Let $x$ and 


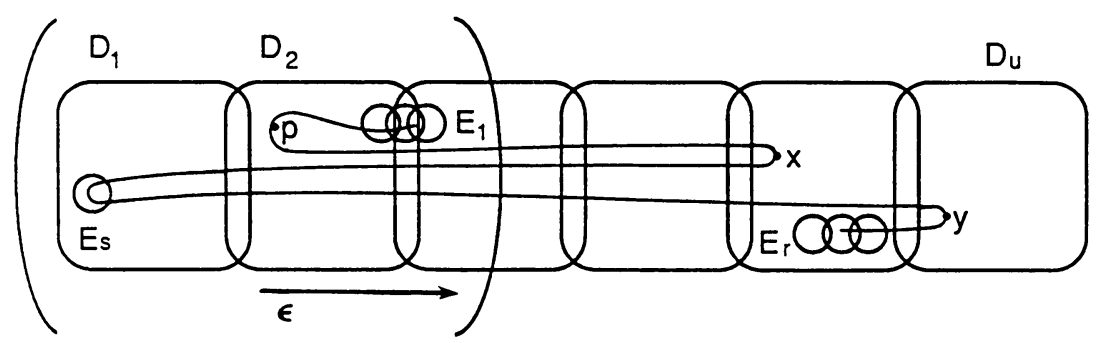

FIGURE 5

$y$ be points of $X-B(p, \varepsilon)$. Then if $E_{1}, \ldots, E_{r}$ is another $\delta$-chain of $X$, then its subchain from $x$ to $y$ contains no link which is contained in $D_{1}-D_{2}$.

Proof. Suppose to the contrary that $E_{s}$ is a link of the subchain from $x$ to $y$ which is contained in $D_{1}-D_{2}$. Let $L=\bigcup_{j=1}^{s} E_{j}$ and $R=\bigcup_{j=s}^{r} E_{j}$. Let $m$ be the greatest integer such that $D_{m}$ intersects $L$, and let $n$ be the greatest integer such that $D_{n}$ intersects $R$. Then $D_{m} \cap L, D_{m-1} \cap L, \ldots, D_{1} \cap L, D_{1} \cap R$, $D_{2} \cap R, \ldots, D_{n} \cap R$ is a $\delta$-chain of $X$ which contradicts condition (7).

Step 2. Suppose condition (7) is satisfied. Then for each positive number $\varepsilon$, there is an open set $U$ and a positive number $\delta^{\prime}$ such that: if $x$ and $y$ are points in the complement of $B(p, \varepsilon)$, and $E_{1}, \ldots, E_{r}$ is any $\delta^{\prime}$-chain of $X$, then the subchain of the $E$ 's from $x$ to $y$ misses $U$.

PROOF. Let $\varepsilon$ be given, and let $\delta$ be a number as guaranteed by Condition (7). Let $D_{1}, \ldots, D_{u}$ be a specific $\delta / 3$-chain of $X$ with $p \in D_{k}$ and $\bigcup_{j=1}^{k} D_{j}$ a subset of $B(p, \varepsilon)$. Choose $U=D_{1}$, and let $\delta^{\prime}$ be a Lebesgue number of this chain. Apply Step 1 to the $\delta$-chain $D_{1} \cup D_{2} \cup D_{3}, D_{4}, \ldots, D_{u}$. Then if $x$ and $y$ are points of the complement of $B(p, \varepsilon)$, and $E_{1}, \ldots, E_{r}$ is a $\delta^{\prime}$-chain of $X$, the subchain between $x$ and $y$ misses $U$. For if a link $E_{s}$ intersected $U\left(=D_{1}\right)$, then by use of the Lebesgue number, $E_{s}$ would be contained in $D_{1} \cup D_{2}$, which implies that $E_{s}$ is contained in $D_{1} \cup D_{2} \cup D_{3}-D_{4}$, in blatant violation of Step 1 .

Step 3 (Proof OF Proposition 1.11). Assume condition (7), and suppose that $x$ and $y$ are points of $X$ different from $p$. We will show that $X$ is not irreducible between $x$ and $y$.

Let $\varepsilon$ be one third the minimum of the distances from $x$ to $p$ and from $y$ to $p$. Let $U$ and $\delta^{\prime}$ be as in Step 2 (for this given $\varepsilon$ ). Finally, let $C^{1}, C^{2}, C^{3}, \ldots$ be a sequence such that, for each $j, C^{j}$ is a $\delta^{\prime} / j$-chain of $X$, and let $C_{x y}^{j}$ denote the subchain of $C^{j}$ from $x$ to $y$. Notice that, for each $j$, the point set $\left(C_{x y}^{j}\right)^{*}$ of $C_{x y}^{j}$ is contained in $X-U$ (by Step 2). Let $Z=$ the closure of $\bigcup_{j=1}\left(C_{x y}^{j}\right)^{*}$. Now $Z$ contains arbitrarily small chains from $x$ to $y$ (guess which ones), hence there is no separation of $Z$ with $x$ and $y$ in different pieces. Thus, by the Splitting Theorem, $Z$ contains a continuum intersecting (containing) both $x$ and $y$. But $Z$ is contained in $X-U$, so $X$ is not irreducible between $x$ and $y$. Therefore, condition (7) implies condition (1).

This completes the proof of Theorem 1.0. 


\section{REFERENCES}

1. Lida K. Barrett, The structure of decomposable snakelike continua, Duke Math. J. 28 (1961), 515-522.

2. R. H. Bing, A homogeneous indecomposable plane continuum, Duke Math. J. 15 (1948), 729742.

3. __ Concerning hereditarily indecomposable continua, Pacific J. Math. 1 (1951), 43-51.

4. __ Snake-like continua, Duke Math. J. 18 (1951), 653-663.

5. J. G. Hocking and G. S. Young, Topology, Addison-Wesley, 1961.

6. E. E. Moise, An indecomposable plane continuum which is homeomorphic to each of its nondegenerate subcontinua, Trans. Amer. Math. Soc. 63 (1948), 581-594.

7. G. T. Whyburn, Analytic topology, Amer. Math. Soc. Colloq. Publ., vol. 28, Amer. Math. Soc., Providence, R.I., 1942.

Department of Mathematics, University of Wyoming, LaRAmie, Wyoming 